\title{
Specific heat anomalies of open quantum systems
}

\author{
Gert-Ludwig Ingold, ${ }^{1,2, *}$ Peter Hänggi, ${ }^{1}$ and Peter Talkner ${ }^{1}$ \\ ${ }^{1}$ Institut für Physik, Universität Augsburg, D-86135 Augsburg, Germany \\ ${ }^{2}$ Laboratoire Kastler Brossel, CNRS, ENS, UPMC, Campus Jussieu Case 74, F-75252 Paris Cedex 05, France
}

(Received 9 February 2009; published 9 June 2009)

\begin{abstract}
The evaluation of the specific heat of an open damped quantum system is a subtle issue. One possible route is based on the thermodynamic partition function which is the ratio of the partition functions of system plus bath and of the bath alone. For the free damped particle it has been shown, however, that the ensuing specific heat may become negative for appropriately chosen environments. Being an open system this quantity then naturally must be interpreted as the change in the specific heat obtained as the difference between the specific heat of the heat bath coupled to the system degrees of freedom and the specific heat of the bath alone. While this difference may become negative, the involved specific heats themselves are always positive; thus, the known thermodynamic stability criteria are perfectly guaranteed. For a damped quantum harmonic oscillator, instead of negative values, under appropriate conditions one can observe a dip in the difference of specific heats as a function of temperature. Stylized minimal models containing a single oscillator heat bath are employed to elucidate the occurrence of the anomalous temperature dependence of the corresponding specific heat values. Moreover, we comment on the consequences for the interpretation of the density of states based on the thermal partition function.
\end{abstract}

DOI: 10.1103/PhysRevE.79.061105

PACS number(s): 05.30. $-\mathrm{d}, 05.70 .-\mathrm{a}, 65.40 . \mathrm{Ba}, 65.80 .+\mathrm{n}$

\section{INTRODUCTION}

An open classical system in contact with a heat bath can often be modeled in terms of a Langevin dynamics with constant friction and white Gaussian noise sources obeying a fluctuation-dissipation theorem [1-3]. A remarkable feature then is the circumstance that the equilibrium statistics of the open classical system turns out to be independent of the coupling strength between the system and the heat bath. In other words, the canonical equilibrium for a classical damped Langevin dynamics agrees with the canonical equilibrium of the isolated system. This feature is rooted in the fact that in this case the so termed "Hamiltonian of mean force" is still given by the bare system Hamiltonian [4]. In clear contrast, this property in general no longer holds true for open systems in the quantum regime beyond the weak-coupling limit [5]. In particular, the canonical equilibrium state of an open quantum system then typically involves an explicit dependence on the system-bath coupling strength.

Motivated by this fact, the study of the specific heat beyond the weak-coupling limit has recently received considerable attention, in particular in view of the validity of the third law of thermodynamics [6-12]. Apart from fundamental thermodynamical questions the study of the specific heat in the quantum regime is also of interest because it can be related to entanglement properties [13].

Recently, two different routes toward the evaluation of a specific heat were proposed and discussed [6,9]. One possibility is based on the thermal expectation value of the Hamiltonian describing the isolated system. Another approach, on which we will focus in this paper, starts out from the thermodynamic partition function of the dissipative system $[6,9,14-24]$

\footnotetext{
*gert.ingold@physik.uni-augsburg.de
}

$$
Z=\frac{\operatorname{Tr}_{S+B}[\exp (-\beta H)]}{\operatorname{Tr}_{B}\left[\exp \left(-\beta H_{B}\right)\right]},
$$

where the total Hamiltonian

$$
H=H_{S}+H_{B}+H_{S B}
$$

consists of terms describing the system, the bath, and the system-bath coupling, respectively. In the absence of a coupling between system and bath, $Z$ reduces to the partition function of the system. Partition function (1) appears naturally in the Feynman-Vernon approach to dissipative systems $[15,16,18,19]$ and can be related to equilibrium properties of the system $[17,23]$.

From Eq. (1), one obtains by means of standard thermodynamic relations a specific heat [25], reading

$$
C=k_{B} \beta^{2} \frac{\partial^{2}}{\partial \beta^{2}} \ln (Z) \text {. }
$$

Here, $k_{B}$ is the Boltzmann constant and the temperature $T$ appears through $\beta=1 / k_{B} T$.

In the following, we will assume the bath to consist of harmonic oscillators and the coupling to be bilinear in system and bath coordinates [5]. In such a framework Ref. [9] found for the damped free particle that under certain circumstances specific heat (3) can become negative. In the case of the Drude model, for example, where the Laplace transform of the damping kernel is given by

$$
\hat{\gamma}(z)=\frac{\gamma \omega_{D}}{z+\omega_{D}},
$$

the specific heat exhibits negative values at low temperatures if the damping constant $\gamma$ exceeds the cutoff frequency $\omega_{D}$, i.e., $\gamma>\omega_{D}$. This behavior is depicted in Fig. 1. For a general damping kernel, a negative specific heat will appear at low temperatures if $\hat{\gamma}^{\prime}(0)<-1$ (see Ref. [9]). No negative spe- 


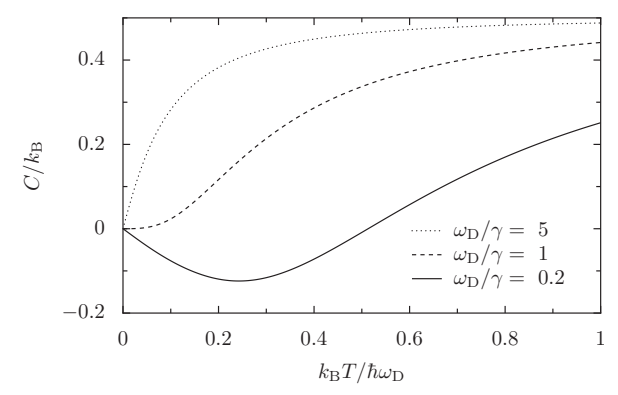

FIG. 1. The specific heat as defined in Eq. (3) for a free damped particle with Drude damping kernel (4) is shown as a function of temperature for $\omega_{D} / \gamma=0.2,1$, and 5. Note that in contrast to Ref. [9], temperature is given in units of the cutoff frequency $\omega_{D}$ instead of the damping strength $\gamma$.

cific heat was found if the free particle is replaced by a harmonic oscillator.

In this paper, we will elucidate the origin of the negative specific heat for the case of the free damped particle and its absence for the damped harmonic oscillator by considering two minimal models. Before doing so, a general comment on the appearance of a negative specific heat is appropriate.

The rationale behind the definition of a specific heat based on thermodynamic partition function (1) is that this partition function should be associated with an effective description of an open system. From this point of view, a negative specific heat may appear disturbing because it raises doubts as to the thermodynamic stability of the system.

However, the meaning of thermodynamic partition function (1) of the open system can be better understood from the point of view of its finite coupling to the heat bath. Specific heat (3) can in fact be expressed as

$$
C=C_{S+B}-C_{B},
$$

where $C_{S+B}$ is the specific heat of system and heat bath while $C_{B}$ is the specific heat of the heat bath alone.

Therefore, $C$ describes the change of the specific heat when the heat bath is enlarged by coupling it to system degrees of freedom. This difference expression in Eq. (5) must be expected on physical grounds when dealing with an open system that is not heat insulated from its environment. For example, take a certain amount of an agent within a container: The established experimental procedure to determine the specific heat of this agent is first to measure the specific heat of the empty container and to subtract this value from the measured specific heat of the combined system to finally arrive at the specific heat of the agent alone. For a macroscopic amount of the agent this value is truly agent specific, i.e., independent of the particular nature of the container and its interaction with the agent (provided the interaction of the agent with the container is short ranged). For a nanoscopic system, however, the energy that is contained in the systembath coupling typically cannot be neglected and consequently will influence the specific heat of such a system. Notably the thermodynamic internal energy of the open system also typically differs from the thermal expectation of the bare system Hamiltonian [4,7]. As a consequence, the values (a)

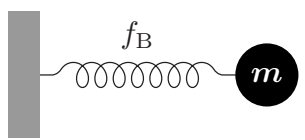

(b)

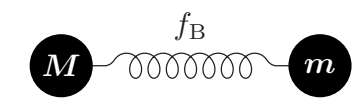

(c)

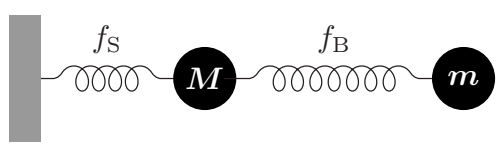

FIG. 2. (a) One single bath oscillator represented by a mass $m$ harmonically coupled to a wall of infinite mass. (b) Bath oscillator coupled to a free system degree of freedom. (c) Bath oscillator coupled to a harmonically bound system degree of freedom. The spring constants of the bath and system oscillator are denoted by $f_{B}$ and $f_{S}$, respectively.

of the specific heat evaluated along such different routes then differ as well $[6,9]$.

The coupling can thus result in a negative specific heat of the open quantum system. However, the involved specific heats, i.e., those of system plus bath on the one hand and of the bath alone on the other hand, are each positive so that no issues concerning the thermodynamic stability arise [26]. Such physical situations are not uncommon as evidenced by recent discussions in the context of the Casimir effect [27], the multichannel Kondo effect [28], or the physics of mesoscopic superconductors that contain magnetic impurities [29].

\section{FREE PARTICLE}

In order to elucidate the appearance of negative specific heat (3) it is sufficient to consider a stylized minimal model where the "bath" consists of only a single degree of freedom described by the Hamiltonian

$$
H_{B}=\frac{p^{2}}{2 m}+\frac{f_{B}}{2} q^{2},
$$

where $f_{B}$ denotes the spring constant. In the following, we will study a system governed by the Hamiltonian

$$
H_{S}=\frac{P^{2}}{2 M}+\frac{f_{S}}{2} Q^{2},
$$

both in the cases of a free particle (spring constant $f_{S}=0$ ) and of a harmonic oscillator $\left(f_{S}>0\right)$. The coupling Hamiltonian is given by

$$
H_{S B}=-f_{B} q Q+\frac{f_{B}}{2} Q^{2},
$$

where the last term renormalizes the potential in order to ensure translational invariance in the case of the free particle. Figure 2 illustrates our two minimal models. The starting point is a single bath oscillator with mass $m$ as depicted in Fig. 2(a). Coupling the system mass $M$ to the bath oscillator leads to the harmonically coupled system of two masses 
shown in Fig. 2(b). If the system degree of freedom corresponds to a harmonic oscillator, we obtain the mechanical system of Fig. 2(c).

It is quite obvious that an environment consisting of one degree of freedom does not suffice to replace any realistic heat bath. In particular, it does not lead to a truly dissipative behavior of the open system to which it couples. Nevertheless, it turns out that even such minimal bath models give rise to the same thermodynamic anomalies that are also encountered with more realistic large environments.

We begin our analysis with a free particle in contact with the single-degree-of-freedom environment described by Eqs. (6) and (8). System and bath are assumed to stay in thermal equilibrium with each other at the inverse temperature $\beta$. Hence, the density matrix of the total system is given by a Gibbs state reading

$$
\rho_{S B}=Z_{S B}^{-1} \exp \left[-\beta\left(H_{S}+H_{B}+H_{S B}\right)\right],
$$

where $Z_{S B}=\operatorname{Tr} \exp \left[-\beta\left(H_{S}+H_{B}+H_{S B}\right)\right]$ denotes the partition function of the total system.

The partition function $Z_{B}=\operatorname{Tr} \exp \left[-\beta H_{B}\right]$ of the isolated bath degree of freedom is given by

$$
Z_{B}=\frac{1}{2 \sinh \left(\frac{\hbar \beta \omega}{2}\right)},
$$

where

$$
\omega=\left(\frac{f_{B}}{m}\right)^{1 / 2}
$$

is the frequency of the bath oscillator. From Eq. (3) the specific heat of this bath follows as

$$
C_{B}=k_{B} g\left(\frac{\hbar \beta \omega}{2}\right)
$$

with the abbreviation

$$
g(x)=\left(\frac{x}{\sinh (x)}\right)^{2} .
$$

If we add the system degree of freedom in order to obtain the mechanical system shown in Fig. 2(b), the partition function contains contributions of two degrees of freedom related to the center-of-mass and the relative motion. The first one is described by a free particle with an effective mass $m+M$ while the second degree of freedom corresponds to a harmonic oscillator with effective mass $m M /(m+M)$ and the frequency

$$
\bar{\omega}=\left(1+\frac{m}{M}\right)^{1 / 2} \omega .
$$

As discussed in Sec. I, negative values of the specific heat occur for the Drude model if the damping strength exceeds the cutoff frequency of the heat bath. Within our minimal model the specific heat may become negative if the mass ratio $m / M$ exceeds a value slightly above 4 . Then, $\bar{\omega}$ is significantly larger than $\omega$, a fact which will be relevant for the discussion of specific heat (16) below.

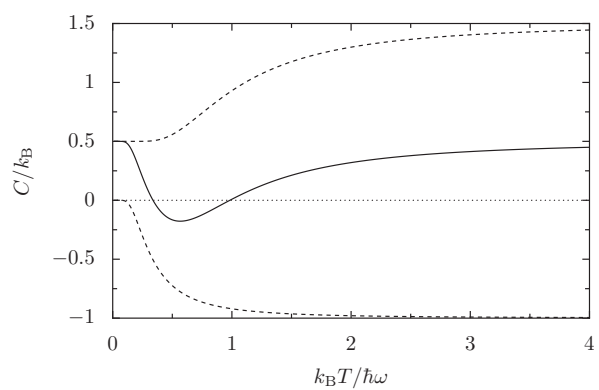

FIG. 3. The difference of specific heats as function of the temperature for the case of a free particle coupled to a single oscillator bath for the mass ratio $m / M=10$. The upper dashed curve corresponds to the first two terms in Eq. (16), i.e., to the specific heat of the system shown in Fig. 2(b) while the lower dashed curve corresponds to the third term in Eq. (16), i.e., to the negative of the specific heat of the bath oscillator shown in Fig. 2(a). The resulting difference is depicted as solid line and displays a temperature region where it takes on negative values.

In order to obtain a well-defined partition function for the free particle, we restrict its motion to a region of length $L$. This length is supposed to be sufficiently large such that the energy-level spacing can be neglected if compared with the thermal energy $k_{B} T$ [9]. Under this condition $L$ will turn out to be irrelevant in the sequel.

The partition function of system plus bath consists of a product of contributions arising from the two normal modes, i.e., the center-of-mass and relative motion, and thus reads

$$
Z_{S B}=\frac{L}{\hbar}\left[\frac{2 \pi(m+M)}{\beta}\right]^{1 / 2} \frac{1}{2 \sinh \left(\frac{\hbar \beta \bar{\omega}}{2}\right)} .
$$

From Eqs. (10), (12), (13), and (15) it is straightforward to evaluate specific heat (3) which becomes

$$
\frac{C}{k_{B}}=\frac{1}{2}+g\left(\frac{\hbar \beta \bar{\omega}}{2}\right)-g\left(\frac{\hbar \beta \omega}{2}\right) \text {. }
$$

The first term arises from a free particle while the second and third term describe the change in specific heat due to the increase in the oscillator frequency from $\omega$ to $\bar{\omega}$ as given by Eq. (14).

In Fig. 3 the contributions to Eq. (16) are sketched. The upper dashed curve corresponds to the first two contributions arising from system and bath. It contains $k_{B} / 2$ from the isolated free particle and a contribution from the harmonic oscillator which is strongly suppressed at low temperatures and reaches $k_{B}$ for high temperatures. The lower dashed curve corresponds to the third term in Eq. (16). The main point to note is the relative shift in temperature of the two contributions due to the change of the oscillator frequency. In the presence of the system, the oscillator frequency is increased according to Eq. (14). As a consequence, there is a temperature window, where the specific heat of system and bath is already significantly suppressed while this is not yet the case for the bath oscillator alone. In this regime, difference (16) of the specific heats can become negative. Note that in contrast to Fig. 1 this temperature window does not extend all the 


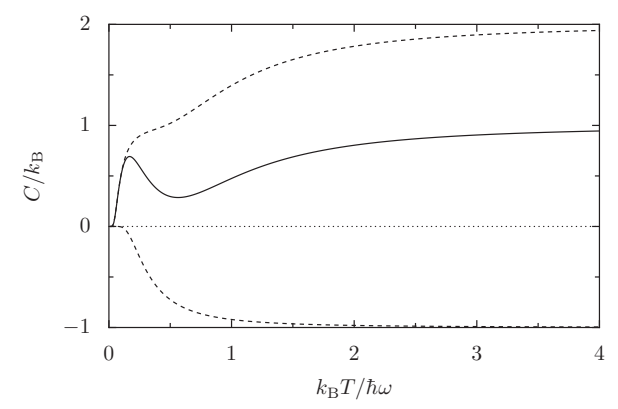

FIG. 4. The difference of specific heats as function of the temperature for the case of a harmonic oscillator coupled to a single oscillator bath. The upper dashed curve corresponds to the first two terms in Eq. (18), i.e., to the specific heat of the system shown in Fig. 2(c) while the lower dashed curve corresponds to the third term in Eq. (18), i.e., to the negative of the specific heat of the bath oscillator shown in Fig. 2(a). The resulting difference is depicted as solid line and displays a dip for the chosen parameter values $\Omega$ $=\omega$ and $m / M=10$.

way down to zero temperature. This is explained by the fact that the bath consists of only one oscillator so that the lowfrequency oscillators present in Drude model (4) on which Fig. 1 is based are missing.

\section{HARMONIC OSCILLATOR}

After having explained the origin of a negative specific heat for a free particle in contact with an environment we will now address the question why such a scenario cannot be realized for a harmonic oscillator.

\section{A. Minimal environment}

To this end, we determine the difference of specific heats for the mechanical systems shown in Figs. 2(a) and 2(c). The system frequency associated with the spring constant $f_{S}$ is given by $\Omega=\left(f_{S} / M\right)^{1 / 2}$. The eigenfrequencies of system plus bath are readily obtained as

$$
\begin{aligned}
\omega_{ \pm}^{2}= & \frac{1}{2}\left(\frac{m+M}{M} \omega^{2}+\Omega^{2}\right) \pm\left[\frac{1}{4}\left(\frac{m+M}{M} \omega^{2}+\Omega^{2}\right)^{2}\right. \\
& \left.-\omega^{2} \Omega^{2}\right]^{1 / 2} .
\end{aligned}
$$

One can show that $\omega_{-} \leq \omega \leq \omega_{+}$, where $\omega_{-}=\omega$ or $\omega_{+}=\omega$ for $m=0$, depending on whether $\omega / \Omega>1$ or $\omega / \Omega<1$. For increasing mass ratio $m / M$ and fixed frequency ratio $\omega / \Omega$ both gaps from $\omega$ to $\omega_{-}$and $\omega_{+}$widen. In the limit $m \gg M$, at any fixed frequency ratio $\omega / \Omega$ one finds $\omega_{-}=(M / m)^{1 / 2} \Omega$ and $\omega_{+}=(m / M)^{1 / 2} \omega$.

From Eqs. (1) and (3) one then obtains

$$
\frac{C}{k_{B}}=g\left(\frac{\hbar \beta \omega_{+}}{2}\right)+g\left(\frac{\hbar \beta \omega_{-}}{2}\right)-g\left(\frac{\hbar \beta \omega}{2}\right),
$$

where $k_{B} g$ is the specific heat of a harmonic oscillator defined in Eqs. (12) and (13). A typical scenario for the case of sufficiently well-separated frequencies is sketched in Fig. 4.
Although for the harmonic oscillator a dip in the specific heat may appear, no negative values can be obtained. The main difference to the case of a free particle is the specific heat of the isolated system degree of freedom: While for not too low temperatures the specific heat for the harmonic oscillator equals $k_{B}$ it is only half as large for the free particle. The difference of the specific heats of the bath oscillator in the presence and absence of the system degree of freedom may reach values up to $k_{B}$, thereby opening up the possibility of negative values of the specific heat for the free particle but not for the harmonic oscillator.

\section{B. Drude bath}

The dip in the specific heat of the harmonic oscillator in contact with the minimal environment can also be observed for a bath giving rise to ohmic damping, i.e., where $\hat{\gamma}(0)$ $>0$. For the ratio of partition functions (1) one finds

$$
Z=\frac{1}{\hbar \beta \Omega} \prod_{n=1}^{\infty} \frac{\nu_{n}^{2}}{\nu_{n}^{2}+\nu_{n} \hat{\gamma}\left(\nu_{n}\right)+\Omega^{2}},
$$

where $\nu_{n}=2 \pi n / \hbar \beta$ are the Matsubara frequencies.

We specifically consider a Drude model characterized by damping kernel (4) with a cutoff frequency $\omega_{D}$. Introducing the quantities

$$
\Lambda_{D}=\frac{\hbar \beta \omega_{D}}{2 \pi}
$$

and

$$
\Lambda_{i}=\frac{\hbar \beta \lambda_{i}}{2 \pi}, \quad i=1,2,3
$$

with

$$
x^{3}+\omega_{D} x^{2}+\left(\gamma \omega_{D}+\Omega^{2}\right) x+\omega_{D} \Omega^{2}=\left(x-\lambda_{1}\right)\left(x-\lambda_{2}\right)\left(x-\lambda_{3}\right),
$$

we obtain from Eqs. (3) and (19)

$$
\begin{aligned}
\frac{C}{k_{B}}= & 1+\Lambda_{1}^{2} \psi^{\prime}\left(1-\Lambda_{1}\right)+\Lambda_{2}^{2} \psi^{\prime}\left(1-\Lambda_{2}\right)+\Lambda_{3}^{2} \psi^{\prime}\left(1-\Lambda_{3}\right) \\
& -\Lambda_{D}^{2} \psi^{\prime}\left(1-\Lambda_{D}\right) .
\end{aligned}
$$

Here, $\psi^{\prime}(x)$ denotes the trigamma function. Specific heat (23) is shown in Fig. 5 for $\gamma=5 \Omega$ and $\omega_{D}=0.1 \Omega$, where $\Omega$ is the frequency of the system oscillator. In contrast to the undamped case, the specific heat increases linearly with temperature with a slope proportional to the damping constant. Then, for sufficiently small cutoff frequency the specific heat goes through a dip at low temperatures before it asymptotically approaches its high-temperature value $k_{B}$.

\section{DENSITY OF STATES}

One can use partition function (1) to formally define an effective density of states $\rho(E)$ of the system by means of the relation [21] 


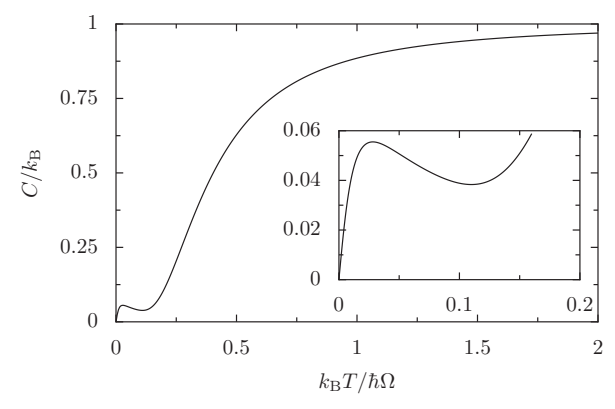

FIG. 5. Specific heat (23) of a damped harmonic oscillator for Drude damping with $\gamma=5 \Omega$ and $\omega_{D}=0.1 \Omega$. The inset shows a close-up of the low-temperature regime.

$$
Z=\int_{0}^{\infty} d E \rho(E) \exp (-\beta E)
$$

For the free particle, it was found in Ref. [9] that the density of states obtained from Eq. (24) can become negative for appropriately chosen environments, e.g., a Drude model with $\omega_{D}<\gamma$. For an environment consisting of a single bath mode like in the minimal models discussed in Secs. II and III it was demonstrated that the appearance of negative contributions to the effective density of states is generic. In particular, the minimal model for a harmonic oscillator coupled to an environmental mode displayed in Fig. 2(c) leads to negative delta functions in the density of states (see Ref. [9]). This is in contrast to our finding for the specific heat which remains always positive for the harmonic oscillator. Interestingly, for a heat bath exhibiting a continuous distribution of bath oscillators, one obtains again a positive density of states despite of the fact that dips in the specific heat like the one shown in Fig. 5 can be observed. Figure 6 displays the density of states for the same parameters as employed in Fig. 5.

An important difference between the specific heat and the density of states as defined by Eq. (24) lies in the fact that the latter cannot be interpreted in terms of a difference of two densities of states. We recall that such an interpretation was possible for the specific heat only because according to

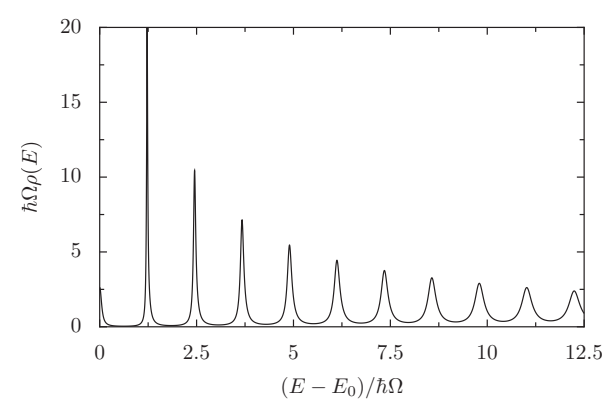

FIG. 6. Density of states (24) of a damped harmonic oscillator for Drude damping with $\gamma=5 \Omega$ and $\omega_{D}=0.1 \Omega$. For these parameters, Eq. (22) yields a resonance frequency of about 1.224 $\Omega$. A delta function contribution of weight one present at the ground-state energy $E_{0}$ of the damped harmonic oscillator is not shown [30].
Eq. (3) it depends linearly on the logarithm of partition function (1). The specific heat shares this property with other thermodynamic quantities like the internal energy and the free energy. The absence of a logarithm of the partition function in Eq. (24) indicates that the effective density of states does not lend itself to an interpretation in terms of the difference of two densities of states. Despite the fact that the effective density of states of a damped harmonic oscillator in the weak-coupling limit, i.e., when the damping strength represents the smallest frequency scale, displays resonances at the expected energies and even yields the correct level widths [23], it therefore remains unclear whether the meaning of the effective density of states goes beyond that of a merely formal notion.

\section{CONCLUSIONS}

For a free damped particle, the specific heat based on effective partition function (1) can become negative. We have demonstrated that this surprising behavior does not endanger the thermodynamic stability of the damped system. Instead, the specific heat should be interpreted as the change in the specific heat of the environment when a system degree of freedom is attached to it. For a damped harmonic quantum oscillator, the difference of specific heats cannot become negative but may display a dip instead. The difference in the behavior of the free particle and the harmonic oscillator can be traced back to the specific heat of the uncoupled system which for the free particle is smaller by a factor of two. All those quantities that are obtained from the logarithm of the partition function by means of a linear operation can be interpreted as differences between the corresponding quantities of the total system plus bath and of the bath alone. This reasoning though does not apply to the density of states which is the inverse Laplace transform of the partition function itself [see Eq. (24)]. By this transformation the ratio of two partition functions yields a complex quantity that obviously cannot be interpreted as a difference of the densities of states of the total system and of the environment.

\section{ACKNOWLEDGMENTS}

One of us (G.-L.I.) is indebted to Hermann Grabert for a discussion which motivated the present work. The manuscript was completed during a stay of G.-L.I. at the Laboratoire Kastler Brossel with financial support by the European Science Foundation (ESF) within the activity "New Trends and Applications of the Casimir Effect" (www.casimirnetwork.com). This work was supported by DFG via research center SFB-486, project A10, and the German Excellence Initiative via the Nanosystems Initiative Munich (NIM). 
[1] W. T. Coffey, Yu. P. Kalmykov, and J. T. Waldron, The Langevin Equation (World Scientific, Singapore, 2004).

[2] R. Zwanzig, Nonequilibrium Statistical Mechanics (Oxford University Press, Oxford, 2001).

[3] P. Hänggi, P. Talkner, and M. Borkovec, Rev. Mod. Phys. 62, 251 (1990).

[4] M. Campisi, P. Talkner, and P. Hänggi, Phys. Rev. Lett. 102, 210401 (2009).

[5] P. Hänggi and G.-L. Ingold, Chaos 15, 026105 (2005).

[6] P. Hänggi and G.-L. Ingold, Acta Phys. Pol. B 37, 1537 (2006).

[7] C. Hörhammer and H. Büttner, J. Stat. Phys. 133, 1161 (2008).

[8] M. Bandyopadhyay, J. Stat. Mech.: Theory Exp. (2009) P05002.

[9] P. Hänggi, G.-L. Ingold, and P. Talkner, New J. Phys. 10, 115008 (2008).

[10] C.-Y. Wang and J.-D. Bao, Chin. Phys. Lett. 25, 429 (2008).

[11] J. Kumar, P. A. Sreeram, and S. Dattagupta, Phys. Rev. E 79, 021130 (2009).

[12] M. Bandyopadhyay and S. Dattagupta, e-print arXiv:0903.2952.

[13] M. Wieśniak, V. Vedral, and Č. Brukner, Phys. Rev. B 78, 064108 (2008).

[14] R. P. Feynman and F. L. Vernon, Ann. Phys. (N.Y.) 24, 118 (1963).

[15] R. P. Feynman, Statistical Mechanics (Addison-Wesley, Read- ing, MA, 1972), p. 82.

[16] A. O. Caldeira and A. J. Leggett, Ann. Phys. (N.Y.) 149, 374 (1983).

[17] H. Grabert, U. Weiss, and P. Talkner, Z. Phys. B 55, 87 (1984).

[18] A. J. Leggett, S. Chakravarty, A. T. Dorsey, M. P. A. Fisher, A. Garg, and W. Zwerger, Rev. Mod. Phys. 59, 1 (1987).

[19] H. Grabert, P. Schramm, and G.-L. Ingold, Phys. Rep. 168, 115 (1988).

[20] G. W. Ford, J. T. Lewis, and R. F. O'Connell, Ann. Phys. (N.Y.) 185, 270 (1988).

[21] A. Hanke and W. Zwerger, Phys. Rev. E 52, 6875 (1995).

[22] T. Dittrich, P. Hänggi, G.-L. Ingold, B. Kramer, G. Schön, and W. Zwerger, Quantum Transport and Dissipation (Wiley, New York, 1998), Chap. 4.

[23] G.-L. Ingold, Lect. Notes Phys. 611, 1 (2002).

[24] G. W. Ford and R. F. O’Connell, Phys. Rev. B 75, 134301 (2007).

[25] In Ref. [9] this quantity was referred to as $C^{Z}$.

[26] H. B. Callen, Thermodynamics and an Introduction to Thermostatics, 2nd ed. (Wiley, New York, 1985), Chap. 8.

[27] J. S. Høye, I. Brevik, J. B. Aarseth, and K. A. Milton, Phys. Rev. E 67, 056116 (2003).

[28] S. Florens and A. Rosch, Phys. Rev. Lett. 92, 216601 (2004).

[29] R. Žitko and T. Pruschke, Phys. Rev. B 79, 012507 (2009).

[30] See Ref. [23] for details on the ground-state energy of the damped harmonic oscillator. 